\title{
IDENTIFICATION ET COMMANDE D'UN GROUPE VARIATEUR DE VITESSE : UNE APPROCHE INTER-DISCIPLINAIRE
}

\author{
Serge Bouter ${ }^{1}$, Rachid Malti ${ }^{1,2}$, Camille Armand ${ }^{1}$ \\ Université de Bordeaux \\ ${ }^{1}$ IUT Bordeaux 1, 15 rue Naudet 33175 Gradignan Cedex, France \\ ${ }^{2}$ IMS UMR 5218 CNRS, 351, cours de la Libération - F 33405 Talence cedex-France \\ \{serge.bouter, rachid.malti, camille.armand\}@u-bordeauxl.fr
}

\begin{abstract}
RESUME : La formation des techniciens supérieurs en Génie Electrique et Informatique Industrielle (GEII) est dispensée dans des Instituts Universitaires de Technologie (IUT). Cette formation, qui correspond à une deuxième année de Licence, est validée par le Diplôme Universitaire de Technologie (DUT). Ce diplôme permet aux titulaires de s'insérer dans le monde professionnel ou, pour certains d'entre eux, d'effectuer une poursuite d'études. La formation se déroule sur quatre semestres intitulés $\mathrm{S} 1\left(1^{\circ}\right.$ semestre), S2, S3 et S4 et est découpée en modules. Ce dispositif conduit les étudiants à attribuer une spécifité à leur diplôme à partir des modules qu'ils ont choisis en accord avec leur projet professionnel. L'expérience d'enseignement présentée dans cet article concerne des étudiants qui souhaitent effectuer une poursuite d'études vers le Master. Elle met en œuvre des modules d'enseignement d'informatique industrielle et de mathématiques, organisés sous la forme de cours, de travaux dirigés et pratiques. Elle requiert aussi de la part des étudiants un certain "goût" pour les matières théoriques.
\end{abstract}

Mots clés : Informatique industrielle, mathématiques, moindres carrés, identification, commande numérique.

\section{Présentation du contexte}

\section{A. Nature des parcours proposés en deuxième année}

Aujourd'hui, qu'ils souhaitent une insertion professionnelle ou une poursuite d'études vers d'autres formations de l'enseignement supérieur, les étudiants du DUT GEII [1] doivent construire leur parcours à partir des modules proposés dans leur département et orienter leur parcours professionnel.

Ainsi, le département GEII de l'IUT de Bordeaux offre, au semestre 4, quatre possibilités de parcours. Chaque possibilité approfondit un thème à partir des modules complémentaires établis par le Programme Pédagogique National (PPN) [1]. On peut distinguer quatre colorations correspondant respectivement à quatre groupes de 24 élèves :

- Énergies renouvelables,

- Électronique pour les transmissions,

- Réseaux industriels,

- Poursuites d'études.

C'est ce dernier groupe qui est concerné par l'expérience d'enseignement présentée dans cet article. Si cette expérience s'appuie pour une bonne part sur l'enseignement de l'informatique industrielle, celui des mathématiques y joue un rôle important.

\section{B. Enseignement de l'informatique industrielle}

Une partie de l'enseignement de l'informatique industrielle au sein du département permet aux étudiants de se familiariser avec les aspects logiciels et matériels des microcontrôleurs. D'un point de vue pratique, cet enseignement utilise le plus souvent des cartes cibles équipées du microcontrôleur étudié et des cartes périphériques permettant aux étudiants de se familiariser avec les entrées et les sorties. La maquette pédagogique développée permet d'aborder toutes les bases de l'enseignement des microcontrôleurs et de mettre en œuvre les périphériques les plus courants : entrées tout ou rien (interrupteurs), sorties tout ou rien (LED), clavier matriciel, afficheur LCD, entrées analogiques, liaisons série et parallèle, etc. Des dispositifs annexes sont associés à cette maquette pour réaliser des projets spécifiques.

Les thèmes traités en informatique au semestre 3 et en partie au semestre 4 sont identiques pour tous les groupes. La dernière partie du semestre 4 est consacrée à des projets qui mettent l'accent sur la spécificité du parcours choisi.

Ainsi, par exemple, le groupe «Électronique pour les transmissions » traite un sujet mettant en œuvre le filtrage numérique. 
Quant au groupe «Poursuite d'études », il traite un sujet qui requiert un certain «goût» pour les mathématiques. Par ailleurs, ce groupe doit suivre des modules complémentaires de mathématiques, ce qui le pré-dispose à ce type de sujets.

\section{Problème Étudié et APPROChe INTER-DisCiPLINAIRE}

\section{A. Description du problème}

La commande d'un groupe de variation de vitesse, constitué d'un moteur à courant continu et d'un hacheur, passe par une phase de modélisation.

Dans le cadre des enseignements d'automatique du semestre 3, l'identification de systèmes s'appuie sur des méthodes graphiques temporelles ou fréquentielles. Après avoir choisi la structure d'un modèle linéaire à temps continu, on procède à l'estimation paramétrique de ce dernier. Suite à cette identification, un régulateur est synthétisé, selon un cahier des charges.

Cette même procédure est aujourd'hui appliquée en vue de la synthèse d'une commande par calculateur. L'identification à temps discret y est traitée par les moindres carrés, méthode rarement abordée au niveau DUT.

\section{A. Organisation des enseignements associés au projet}

Ce projet est constitué de deux parties, l'une «théorique» et l'autre «pratique», et occupe des créneaux d'enseignement de mathématiques et d'informatique industrielle.

Dans la partie «théorique », l'algorithme des moindres carrés classique est abordé avec l'enseignant de mathématiques à partir d'un exercice. Après présentation $\mathrm{du}$ critère quadratique, l'enseignant demande aux étudiants :

- de retrouver, à partir d'un ensemble de questions,

l'algorithme ;

- d'appliquer cet algorithme sur un ensemble réduit de mesures.

Cette partie requiert une séance de TD de 1 h30. Suite à cet exercice, l'enseignant de mathématiques utilise le calcul matriciel comme moyen de traitement de l'algorithme des moindres carrés. Ce développement occupe 1 séance de TD supplémentaire.

La partie «pratique » du projet est constituée de quatre phases :

- programme d'acquisition de mesures,

- identification du système,

- synthèse du correcteur,

- programmation de la commande du moteur à courant continu.

Elle requiert 4 séances de 3 heures durant lesquelles les étudiants travaillent en binôme au sein d'un groupe de TP.
III. PRÉ-REQUIS DU GROUPE « POURSUITE D'ÉTUDE »

Au semestre 3, les étudiants suivent un enseignement d'automatique à temps continu où ils abordent :

- les systèmes bouclés,

- l'analyse fréquentielle et temporelle des systèmes,

- la marge de phase et la marge de gain,

- les correcteurs et leurs fonctions,

- $\quad$...

Par ailleurs, l'informatique industrielle permet d'exposer les éléments nécessaires au projet, à savoir :

- les interruptions,

- les entrées/sorties Tout Ou Rien (TOR),

- la liaison série,

- la conversion analogique-numérique,

- la modulation de largeur d'impulsion (PWM pour Pulse Width Modulation),

- $\quad$...

Le groupe « Poursuite d'étude » n'intègre pas dans son parcours l'étude de la commande numérique. Cependant, il connaît, pour l'avoir abordé en mathématiques et en électronique, la transformée en $\mathrm{Z}$ et les équations récurrentes. Il bénéficie au semestre 4 de modules de mathématiques supplémentaires qui permettent de traiter la partie «théorique » du projet.

Ce projet doit montrer certains outils mathématiques utilisés en automatique à des étudiants dont le parcours est orienté vers des études longues. En effet, la partie automatique à temps discret sera éventuellement étudiée et approfondie dans leur futur cursus universitaire.

\section{Didactisation de L'ALGORITHME DES « MoINDRES CARRÉS 》}

L'algorithme des «moindres carrés », appliqué sur un système du premier ordre, est étudié selon deux approches différentes, la première basée sur la notation scalaire et la deuxième sur la notation matricielle.

Les conditions de validité de cet algorithme (excitation persistante, bruit de mesure blanc et de valeur moyenne nulle, estimation paramétrique biaisée en présence de bruit, ...) ne sont pas évoquées. Ces aspects sont généralement vus au niveau Master.

\section{A. Formulation du problème de minimisation de l'erreur quadratique}

Le système mis à la disposition des étudiants (Fig. 1) est constitué d'un hacheur et d'un moteur à courant continu. Ce dispositif est piloté à partir du microcontrôleur 68HC12. L'objectif, dans un premier temps est d'identifier ce système en se fixant une structure de modèle à temps discret linéaire du premier ordre, décrite par l'équation de récurrence suivante :

$$
y_{k}^{M}=a \cdot y_{k-1}^{M}+b \cdot u_{k-1}
$$


où $u_{k}$ et $y_{k}^{M}$ sont respectivement l'entrée et la sortie du modèle à l'échantillon $k$ la période d'échantillonnage étant fixé à $T e$.

L'erreur de sortie à l'échantillon $k$ s'écrit :

$$
e_{k}=y_{k}-\hat{y}_{k}
$$

soit en remplaçant $\hat{y}_{k}$ par son expression :

$$
e_{k}=y_{k}-\left(\frac{b q^{-1}}{1-a q^{-1}}\right) u_{k}
$$

où $q^{-1}$ représente l'opérateur retard. La minimisation de la norme quadratique de l'erreur de sortie (équation précédente) aurait nécessité l'implantation d'un algorithme à base du gradient; La présentation d'un tel algorithme n'est pas envisageable au niveau DUT.

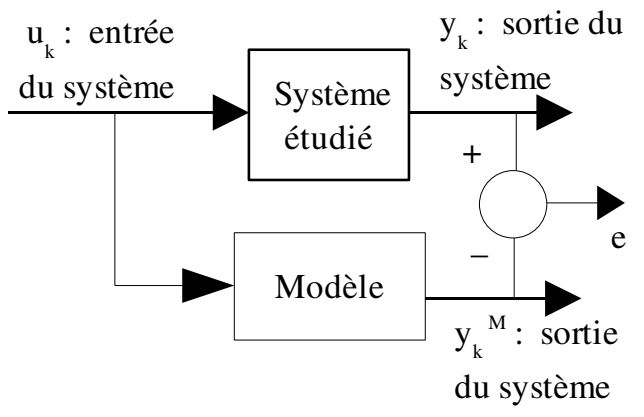

Fig. 1 : Système d'entrée/sortie

C'est pourquoi, il est préférable de définir l'erreur d'équation, aussi appelée erreur de modèle [2] :

qui peut aussi s'écrire :

$$
\epsilon_{k}=\left(1-a q^{-1}\right) e_{k}=\left(1-a q^{-1}\right) y_{k}-b q^{-1} u_{k}
$$

$$
\epsilon_{k}=y_{k}-a y_{k-1}-b u_{k-1}
$$

L'objectif ici est de minimiser la norme quadratique de l'erreur d'équation sur les $K$ mesures d'entrée-sortie.

$$
\|\epsilon\|(a, b)=\sqrt{\frac{1}{K} \sum_{k=1}^{K}\left(y_{k}-a y_{k-1}-b u_{k-1}\right)^{r}}
$$

Puisque la fonction quadratique est croissante, minimiser le carré de la norme quadratique revient à minimiser la norme quadratique elle-même :

$$
J(a, b):=\|\epsilon\|^{2}(a, b)=\frac{1}{K} \sum_{k=1}^{K}\left(y_{k}-a y_{k-1}-b u_{k-1}\right)^{2}
$$

Les notations $J(a, b)$ et $\|\epsilon\|(a, b)$ sont préférées afin de montrer que l'erreur quadratique est une fonction de deux variables $a$ et $b$. Le calcul du minimum de $J$ conduit à l'évaluation des paramètres $a$ et $b$ optimaux, par la méthode des moindres carrés, étudiée selon deux approches différentes, la première basée sur la notation scalaire et la deuxième sur la notation matricielle.

\section{B. Première approche : notation scalaire}

Cette première approche s'intègre dans la progression mathématique en proposant une synthèse de l'étude de fonctions à plusieurs variables (ici 2).

En effet, pour minimiser $J(a, b)$, il est nécessaire d'étudier la dérivée de $J$ par rapport à $a$ et à $b$. Ainsi, les étudiants doivent :

- exprimer $\frac{d J}{d a}$ et $\frac{d J}{d b}$,

- montrer, qu'en posant $\frac{d J}{d a}=\cdot$ et $\frac{d J}{d b}=0$, ils obtiennent un système de deux équations linéaires de la forme :

$$
\begin{aligned}
& a \cdot \alpha+b \cdot \beta=\chi \\
& a \cdot \beta+b \cdot \lambda=\delta
\end{aligned}
$$

- donner les expressions de $\alpha, \beta, \chi, \lambda$ et $\delta$ en fonction des entrées $u_{k}$ et des sorties $y_{k}$, $k=1,2, \ldots K$.

\section{Exercice « simple » d'application}

Un tableau de mesures est fourni aux étudiants correspondant à la réponse d'un système linéaire du

\begin{tabular}{|c|c|c|c|c|c|c|c|}
\hline$k$ & $y_{k}$ & $u_{k}$ & $y_{k-1}{ }^{2}$ & $u_{k-1} y_{k-1}$ & $u_{k-1} u_{k-1}$. & $y_{k} y_{k-1}$ & $y_{k} u_{k-1}$ \\
\hline 0 & 0 & 0 & 0 & 0 & 0 & 0 & 0 \\
\hline 1 & 0 & 100 & 396 & 0 & 0 & 0 & 1991 \\
\hline 2 & 20 & 100 & & & & & \\
\hline 3 & 35 & 100 & & & & & \\
\hline 4 & 47 & 100 & & & & & \\
\hline 5 & 58 & 100 & & & & & \\
\hline 6 & 64 & 100 & & & & & \\
\hline 7 & 70 & 100 & & & & & \\
\hline 8 & 73 & 100 & & & & & \\
\hline 9 & 78 & 100 & & & & & \\
\hline \multicolumn{3}{|c|}{ somme/9 } & $\alpha$ & $\beta$ & $\lambda$ & $x$ & $\delta$ \\
\hline
\end{tabular}
premier ordre à une entrée de type échelon de Heaviside.

Ce tableau se résume à une dizaine de points pour que les étudiants puissent appliquer l'algorithme avec une calculatrice ou en utilisant un tableur. Pour dérouler les calculs le plus facilement possible, les étudiants remplissent le tableau où ils retrouvent les relations mises en évidence précédemment.

Tableau 1: Application de l'algorithme des moindres carrés

A partir des valeurs calculées au tableau 1, les étudiants déterminent les coefficients $a$ et $b$ en résolvant le système d'équations (3)-(4) et expriment la fonction de transfert en $\mathrm{Z}$ associée au modèle de la Fig. 1. 
Enfin, une validation du modèle est effectuée en superposant la réponse du modèle à celle du système sur l'échelon qui a servi à l'identification. De plus, les étudiants constatent que la réponse $\mathrm{du}$ modèle correspond bien à celle du système.

\section{Deuxième approche : notation matricielle}

En complétant l'approche précédente par une formalisation matricielle et la détermination de la nature de l'extremum, cette deuxième approche s'intègre dans la progression mathématique en proposant une transition entre les enseignements du semestre 3 et du semestre 4. Elle permet en effet de reprendre l'étude de fonctions de plusieurs variables et de présenter le calcul matriciel comme un outil de simplification d'écriture.

Comme la tension d'entrée est supposée constante, on pose:

$$
u_{k}=u_{0}, \quad \forall k=1,2, \ldots K
$$

Les paragraphes suivants résument la démarche présentée aux étudiants.

1) Ecriture matricielle de l'erreur d'équation

Posons :

$$
\epsilon=\left(\begin{array}{c}
\epsilon_{1} \\
\ldots \\
\epsilon_{k} \\
\ldots \\
\epsilon_{N}
\end{array}\right) \quad, \quad Y_{0}=\left(\begin{array}{c}
y_{0} \\
\ldots \\
y_{k} \\
\ldots \\
y_{N-1}
\end{array}\right) \quad, \quad Y_{1}=\left(\begin{array}{c}
y_{1} \\
\ldots \\
y_{k} \\
\ldots \\
y_{N}
\end{array}\right) \text { et } \quad U .=\left(\begin{array}{c}
u . \\
\ldots \\
u . \\
\ldots \\
u .
\end{array}\right) \text {. }
$$

D'après l'équation (1), pour $k=1, \ldots, K$, on peut facilement exprimer le vecteur de l'erreur de modèle :

$$
\epsilon=Y,-a Y .-b U \text {. }
$$

Rappelons que l'objectif consiste à déterminer $a$ et $b$ tels que la norme quadratique de l'erreur $\epsilon$, notée $\|\epsilon\|$, soit minimale.

La relation vectorielle (4), peut aussi s'exprimer sous la forme matricielle :

$$
\begin{gathered}
\epsilon=Y_{1}-M p \\
\text { où } M=\left[Y, U_{.}\right]=\left(\begin{array}{cc}
y . & u . \\
\ldots & \ldots \\
y_{N-1} & u .
\end{array}\right) \text { et } p=\left(\begin{array}{l}
a \\
b
\end{array}\right)
\end{gathered}
$$

\section{2) Ecriture matricielle de l'erreur quadratique}

On montre aisément que la notation scalaire de l'erreur quadratique (2), peut s'écrire sous la forme matricielle :

soit, en développant :

$$
\|\epsilon\|^{r}={ }^{t} Y_{1} Y_{1}+{ }^{t} p^{t} M M p-{ }^{t} Y_{1} M p-{ }^{t} p^{t} M Y_{1} .
$$

On remarque que ${ }^{t} p^{t} M Y_{1}$ est un scalaire. Par conséquent :

$$
{ }^{t} p^{t} M Y_{1}={ }^{t}\left({ }^{t} p^{t} M Y_{1}\right)={ }^{t} Y_{1} M p
$$

ou encore,

où

$$
\begin{aligned}
& Q={ }^{t} M M=\left(\begin{array}{cc}
y .{ }^{2}+. .+y_{N-1}{ }^{2} & y . u .+\ldots+y_{N-1} u . \\
y . u .+\ldots+y_{N-1} u . & K u^{r} .
\end{array}\right) \\
& \text { et } S={ }^{t} M Y_{1}=\left(\begin{array}{c}
y . y_{1}+\ldots+y_{N-1} y_{N} \\
u . y_{1}+\ldots+u, y_{N}
\end{array}\right)
\end{aligned}
$$

\section{3) Expression analytique de l'erreur quadratique}

L'erreur quadratique est une fonction à deux variables :

$$
\begin{array}{r}
\|\epsilon\|^{2}: \quad \mathbb{R}^{2} \rightarrow \mathbb{R} \\
(a, b) \rightarrow\|\epsilon\|^{2}(a, b)
\end{array}
$$

En notant $Q=\left(\begin{array}{ll}q_{11} & q_{12} \\ q_{21} & q_{22}\end{array}\right)$ et $S=\left(\begin{array}{l}s_{1} \\ s_{2}\end{array}\right)$, on peut exprimer le carré de l'erreur quadratique de la façon suivante :

$$
\|\epsilon\|^{2}(a, b)=\left\|Y_{1}\right\|^{2}+q_{11} a^{2}+2 q_{12} a b+q_{22} b^{2}-2 a s_{1}-2 b s_{2}
$$

4) Extremum de $\|\epsilon\|^{2}$ grâce à $\quad \overrightarrow{\operatorname{grad}}\left(\|\epsilon\|^{2}\right)$

On a:

$$
\overrightarrow{\operatorname{grad}}\left(\|\epsilon\|^{2}\right)=\left(\begin{array}{c}
\frac{\partial\|\epsilon\|^{2}}{\partial a} \\
\frac{\partial\|\epsilon\|^{2}}{\partial b}
\end{array}\right)=\left(\begin{array}{l}
2 q_{11} a+2 q_{12} b-2 s_{1} \\
2 q_{12} a+2 q_{22} b-2 s_{2}
\end{array}\right)
$$

donc,

$$
\begin{gathered}
\overrightarrow{\operatorname{grad}}\left(\|\epsilon\|^{2}\right)=0 \Leftrightarrow 2 Q p-2 S=0 \\
\overrightarrow{\operatorname{grad}}\left(\|\epsilon\|^{2}\right)=0 \Leftrightarrow Q p=S
\end{gathered}
$$

On attire l'attention des étudiants sur la similitude du système d'équation (3)-(4) et de l'équation matricielle (5).

On s'est ainsi ramené à un système $2 \times 2$, aisément résoluble si $Q$ est inversible par:

$$
\hat{p}=\left(\begin{array}{l}
\hat{a} \\
\hat{b}
\end{array}\right)=Q^{-1} S=\left({ }^{t} M M\right)^{-1 t} M Y
$$

Cette formule peut être utilisée sous Matlab où il est aisé de définir la matrice $M$ et le vecteur $Y$. 
La solution $\hat{p} \quad$ constitue ainsi le vecteur de paramètres pour lequel l'erreur quadratique admet un extremum.

\section{5) Nature de l'extremum $\hat{p}$}

La nature de l'extremum dépend de la matrice Hessienne. Cependant pour les étudiants, on n'aborde pas ces justifications matricielles et on s'en tient au critère de comparaison des trois quantités $r, s$, et $t$ :

$$
\begin{aligned}
& r:=\frac{\partial^{r}\left(\|\epsilon\|^{r}\right)}{\partial a^{r}}(\hat{a}, \hat{b})=r q_{\|} \\
& t:=\frac{\partial^{r}\left(\|\epsilon\|^{r}\right)}{\partial b^{r}}(\hat{a}, \hat{b})=r q_{r r} \\
& s:=\frac{\partial^{r}\left(\|\epsilon\|^{r}\right)}{\partial a \partial b}(\hat{a}, \hat{b})=r_{q_{r}}
\end{aligned}
$$

D'une part, on constate que $r>\cdot$, car $q_{\|}$est une somme de carrés. D'autre part,

$$
\begin{aligned}
r^{2}-s t & =4\left(q_{12}{ }^{2}-q_{11} q_{22}\right) \\
& =4\left(\left(\sum_{0}^{K-1} u_{0} \cdot y_{k}\right)^{2}-\left(\sum_{0}^{K-1} y_{k}{ }^{2}\right)\left(\sum_{0}^{K-1} u_{0}\right)\right)
\end{aligned}
$$

Le théorème de Cauchy-Schwartz assure que

$$
\begin{aligned}
\left(\sum_{\cdot}^{K-1} u_{\cdot} \cdot y_{k}\right)^{r} & <\left(\sum_{\cdot}^{K-1} y_{k}^{r}\right)\left(\sum_{\cdot}^{K-1} u_{\cdot}^{r}\right) \\
& <K u_{\cdot}^{r} \sum_{\cdot}^{K-1} y_{k}^{r}
\end{aligned}
$$

On obtient donc,

$$
r^{r}-s t<
$$

Par conséquent le vecteur $\hat{p}=\left(\begin{array}{c}\hat{a} \\ \hat{b}\end{array}\right)$ correspond bien au minimum de l'erreur quadratique.

\section{ENVIRONNEMENT DE L'EXPÉRIMENTATION}

\section{A. Description du poste de travail}

Le poste de travail, permettant de développer la partie «pratique » du projet, comporte plusieurs ensembles :

- un PC équipé de l'environnement de développement intégré du microcontrôleur utilisé, en l'occurrence le MC68HC12,

- une maquette pédagogique (Fig. 2) incluant les circuits périphériques au microcontrôleur (écran LCD, leds, clavier, ...),
- Un connecteur permettant de récupérer plusieurs broches du microcontrôleur associées à des ports de conversion analogique/numérique et de modulation de largeur d'impulsion.

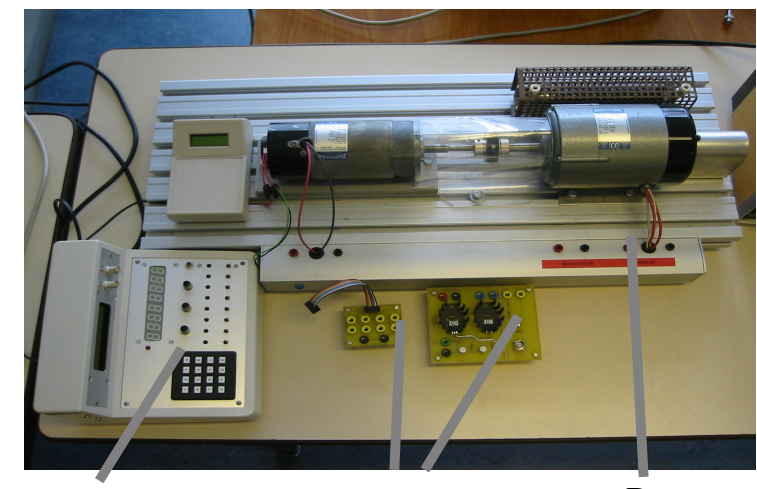

Maquette HC12 Cartes électroniques

Banc moteur

Fig. 2 Dispositif didactique

\section{B. Éléments nécessaires à l'expérimentation}

\section{1) Éléments matériels}

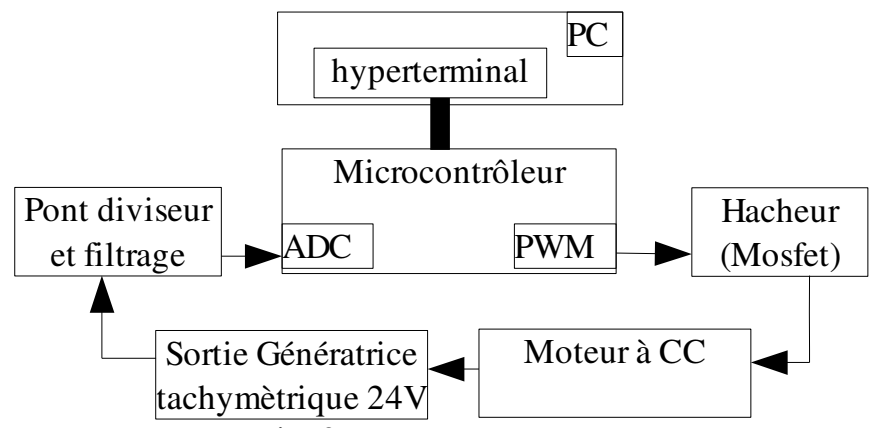

Fig. 3: Synoptique du dispositif

L'induit et l'inducteur du moteur à courant sont alimentés en $24 \mathrm{~V}$. La variation de tension aux bornes de l'induit est obtenue au moyen d'un hacheur série (voir Fig. 2 et Fig. 3). La grille du MOSFET de puissance est commandée par une sortie d'un port PWM (Modulation de Largeur d'Impulsion) du microcontrôleur.

La mesure de la vitesse est effectuée à l'aide d'une génératrice tachymétrique $24 \mathrm{~V}$.

L'interfaçage entre le banc moteur et le microcontrôleur est réalisé au moyen de deux cartes :

- carte de puissance et de conditionnement du signal

« vitesse »,

- carte d'accès aux entrées/sorties des ports du HC12.

\section{2) Éléments logiciels}

Les étudiants ont à leur disposition une bibliothèque de fonctions nécessaires à la réalisation du projet et déjà utilisées au cours de l'année :

- initialisation des ports et de la liaison série,

- émission/réception sur liaison série,

- conversion entier/chaîne de caractère,

- ... 
Ils sont aussi amenés à utiliser «l'hyperterminal» de Windows et le tableur d'OpenOffice.

\section{C. Étude de la bibliothèque de fonctions, des cartes électroniques et du câblage}

A partir des éléments matériels et logiciels à leur disposition, les étudiants proposent un schéma de câblage permettant d'associer les composants du projet :

- cartes électroniques (puissance, mesures, accès aux ports),

- banc moteur (induit, inducteur, génératrice tachymétrique),

- alimentations à courant continu,

- liaison série.

\section{DÉveloppement de L'APPLICATION DESTinée À L'IDENTIFICATION DU SYSTÈME}

\section{A. Programme d'acquisition de données}

On demande aux étudiants de réaliser un programme permettant d'effectuer les opérations suivantes :

- initialiser les ports d'E/S,

- initialiser le timer afin de générer des interruptions selon la période d'échantillonnage de $10 \mathrm{~ms}$,

- démarrer le moteur avec un rapport cyclique de $10 \%$,

- attendre le régime établi,

- accélérer le moteur avec un rapport cyclique de $90 \%$,

- autoriser les interruptions périodiques du Timer et procéder à une mesure à chaque interruption,

- arrêter à partir de la $100^{\text {ème }}$ mesure en inhibant les interruptions,

- transmettre les mesures sur la liaison série.

Pour cela, on propose aux étudiants de reprendre les fonctions de bibliothèques mises à leur disposition pour ce projet. Toutes les notions associées aux opérations que doit exécuter leur application, sont abordées pendant l'année.

Les mesures sont récupérées au moyen de l'application «Hyperterminal » de Windows (Fig.4).

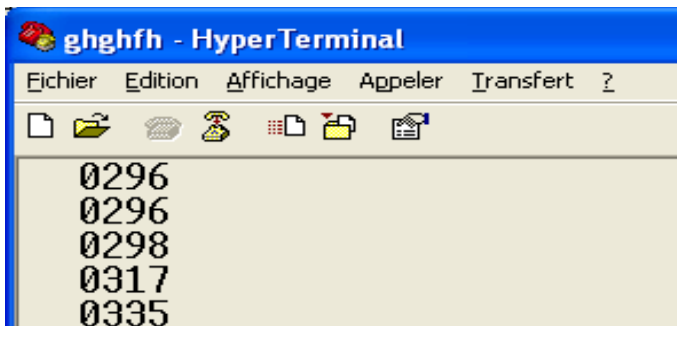

Fig. 4 Récupération des mesures sous l'application "Hyperterminal"

\section{B. Utilisation du tableur pour l'estimation paramétrique}

Les étudiants traitent cette partie au moyen d'un tableur. Ils ont à leur disposition deux feuilles de calcul organisées comme le montre le tableau 1. Ils y recopient les mesures récupérées à partir de l'application «Hyperterminal» (Fig. 5). La deuxième feuille (Fig. 6) affiche les coefficients du système d'équations (3)-(4) permettant de déterminer les paramètres de l'équation récurrente (1).

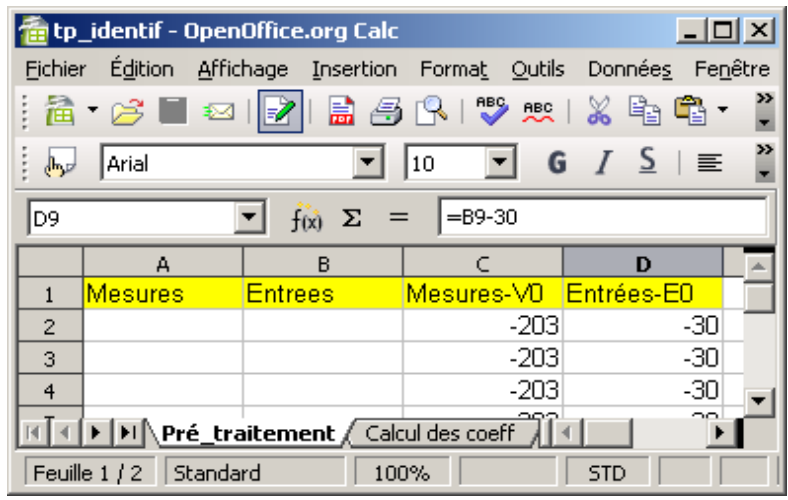

Fig. 5 Première feuille de calcul

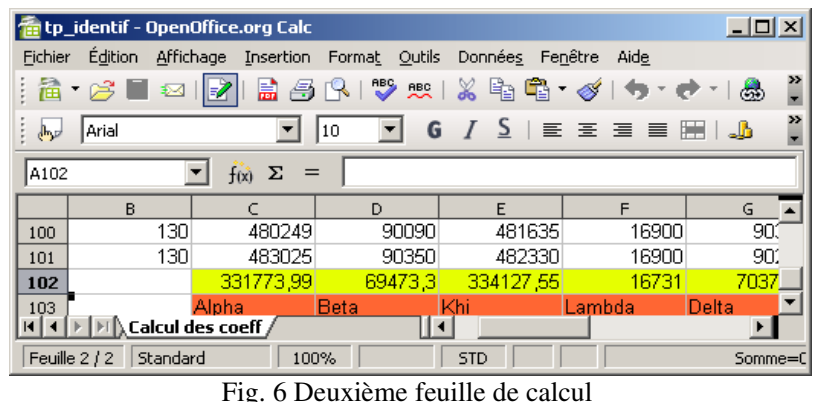

Fig. 6 Deuxième feuille de calcul

\section{VII.DÉVELOPPEMENT DE LA COMMANDE}

A. Synthèse de la loi de commande

\section{1) Remarques préliminaires à la synthèse}

Il est nécessaire de rappeler aux étudiants que :

- la période d'échantillonnage du calculateur (le microcontrôleur $\mathrm{HC12}$ ) cadençant la lecture des entrées, le traitement et l'écriture sur les registres de sortie est identique à celle utilisée pour l'identification,

- le modèle à temps discret déterminé, est seulement valable pour cette période d'échantillonnage,

- la séquence de commande du système suit le chronogramme de la Fig. 7:

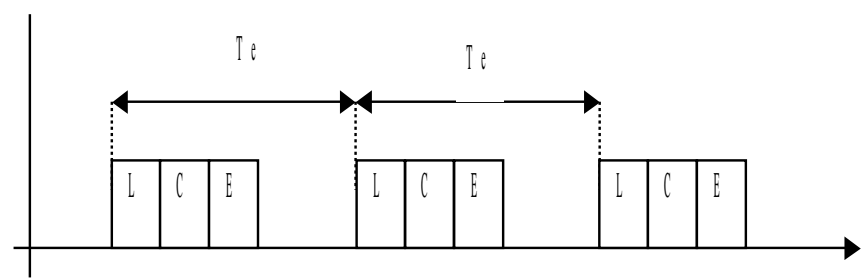

Fig. 7 Séquence d'exécution ; L : lecture du registre d'entrée ; C : calcul associé au correcteur ; E : écriture sur le registre de sortie. 


\section{3) Calcul de la loi de commande}

Le calcul de la loi de commande s'appuie sur le principe de placement de pôles.

On indique aux étudiants que la fonction de transfert en $z$ d'un correcteur Proportionnel Intégral (PI) est de la forme suivante:

$$
C(z)=\frac{c_{0} z-c_{1}}{z-1}
$$

On leur demande :

- de rappeler l'intérêt d'un correcteur PI,

- de donner l'expression de la fonction de transfert en $z$ en boucle fermée, $G(z)$, sous la forme d'une fraction rationnelle,

- de déterminer les paramètres du correcteur $C(z)$, afin que les pôles de $G(z)$ soient $z_{1}=0.8$ et $z_{2}=0.3$, en leur indiquant que ces valeurs permettent d'obtenir une marge de phase raisonnable, supérieure à $45^{\circ}$,

- de donner l'équation récurrente associée et de proposer un algorithme permettant son implantation sur le MC68HC12.

Sur ce dernier point les étudiants doivent :

- implanter une équation récurrente dans une routine

d'interruption périodique,

- choisir le type de variables à utiliser,

- déterminer le domaine de variation de certaines variables,

- borner la commande, de manière à ce que le rapport cyclique de la PWM ne soit pas négatif et ne dépasse pas $100 \%$.

Le microcontrôleur et le compilateur utilisés ne permettent pas d'utiliser facilement les nombres à virgule flottante. Par conséquent, il est préférable de traiter des entiers en travaillant sur le principe des nombres fractionnaires à virgule fixe. On se contente donc d'effectuer des opérations avec des nombres en virgule fixe au moyen de multiplications et de divisions sur des nombres entiers codés sur 8 ou 16 bits. Pour les coefficients décimaux, on utilise un format Qn, obtenu en multipliant le coefficient par $2^{n}$ et en conservant la partie entière.

Exemple sur un format Q5 :

$x=y+2,5 z-1,56 u$

devient :

$$
x=y+(80 z-50 u) / 32
$$

\section{B. Application de régulation}

Un algorithme est présenté aux étudiants afin qu'ils mettent en œuvre les fonctions de régulation de vitesse, de saisie de consigne de vitesse, et d'affichage de la vitesse et de l'erreur.

Cette partie est relativement aisée à implémenter pour les étudiants. En effet il s'agit pour eux de reprendre leur programme d'identification et de l'adapter à cette application de commande.

A partir de quelques essais relativement simples tels que la variations de la tension ou de la consigne, ils constatent une bonne régulation de vitesse.

\section{Conclusions}

Conformément au Programme Pédagogique National DUT GEII, une partie de la formation des étudiants est construite à partir de modules complémentaires leur permettant de définir un parcours en adéquation avec leur Projet Personnel et Professionnel.

Aussi, le département GEII de l'IUT de Bordeaux a organisé le semestre 4 en quatre groupes. L'un de ces groupes a pour vocation de se préparer à la poursuite d'études. Un renforcement en mathématiques et en physique permet de mettre en œuvre des concepts et des outils qui ne sont étudiés généralement qu'à partir de la licence 3. Ainsi, les étudiants de ce groupe ont pu aborder l'algorithme des moindres carrés et réaliser l'implantation d'un correcteur numérique.

Bien que les étudiants de ce groupe ne soient pas directement concernés par l'automatique à temps discret, avec ce projet, ils ont pu constater l'intérêt du «numérique » à partir de :

- la programmation du correcteur,

- la communication entre différentes parties de l'application intégrant la commande numérique,

- la mise en place d'une structure de commande complexe et hiérarchisée.

De plus, la présentation des moindres carrés, pouvant être appliqué à d'autres domaines de la physique, elle permet d'élargir la culture scientifique et technique des étudiants destinés à une poursuite d'études. Les résultats qu'ils ont obtenus sont satisfaisants et démontrent que l'on peut aborder des sujets qui sont habituellement traités en Master.

Il est évident que la mise en place de ces parcours favorise ce type d'expérience et permet l'approfondissement de certains domaines.

\section{BibLIOGRAPHIE}

[1] Programme Pédagogique National du DUT « Génie Électrique et Informatique, septembre 2005

[2] JC Trigeassou, « Recherche de modèles expérimentaux assisté par ordinateur », Lavoisier, 1988.

[3] Commande numérique par placement de pôles, G. Scorletti, G. Binet, E. Pigeon, Université de Caen, 2005 